\title{
VERY WIDE-FIELD MONITORING OF THE SKY
}

\author{
R. HUDEC and J. SOLDÁN \\ Czech Academy of Sciences Astronomical Institute \\ Observatory Ondrejov \\ 251-65 Ondřejov \\ Czech Republic
}

\begin{abstract}
There is a need for very wide-field monitoring of the sky in several branches of astronomy and astrophysics, ranging from meteor research to monitoring of optical transients and counterparts to gamma ray burst sources. The photographic very wide-field monitoring of the sky is run at the Ondrejov Observatory and other stations of the Czech Meteor Network and is sensitive enough to record stars up to $11 \mathrm{mag}$ on 3 - $4 \mathrm{~h}$ exposures or 1-s flashes up to $3.0 \mathrm{mag}$ over a field-of-view of $180 \mathrm{deg}$. diameter. A CCD device for very wide-field monitoring is also in development with sensitivity of 5.0 mag for 1-s flashes (and $10 \mathrm{mag}$ for stars with $100 \mathrm{~s}$ exposure) over a field-of-view of 50 x 60 degrees. Both systems are briefly described and discussed.
\end{abstract}

\section{The Photographic Patrol Network}

There are 10 stations using fish-eye cameras with Opton-Distagon lenses $1: 3.5 \mathrm{f}=3 \mathrm{~cm}$ in the network directed by the Ondfejov Observatory. Eight stations are equipped with stationary cameras, the remaining 2 use both stationary and guided cameras. A typical exposure represents about 4 hours. The photographic plates of the type ORWO NP27 (400 ASA) were used between 1975 and 1993. Planfilms of the type FOMAPAN 100 have been used since 1993 due to a huge increase in the prices of the ORWO plates. The diameter of the visible hemisphere $(2 \pi \mathrm{sr})$ represents $7.5 \mathrm{~cm}$ on the plate. The typical limiting magnitude amounts to $9-11 \mathrm{mag}$ for stars and 3.0-3.5 mag for 1-sec events.

The network operates every clear night except times close to the full moon. The main goal of the network is to detect bright meteors, but there are also general applications in other branches of astrophysics. The main advantages of the network can be summarized as follows:

1) large coverage of the sky both in time and area;

2) multiplicity of the plates taken at different stations allows verification of astrophysical events and exclusion of events occurring in earth atmosphere and near space up to distances of $100,000 \mathrm{~km}$;

3) the sky patrol plates are suitable especially in the:

a) long-term monitoring of bright objects;

b) monitoring of transient phenomena (e.g. optical transients related to gamma ray burst sources); 
c) providing simultaneous ground-based optical data for satellite experiments;

d) monitoring of satellite glints and providing their rates.

The fish-eye cameras have been in use since 1975. A great number of plates $(23,000)$ were taken by driven Tessar cameras (quasi point-like stellar images) in the years 1955-1978. This is valid also for fixed Tessar cameras in 2 stations (46,000 plates). For each camera system, 10 identical cameras with Tessars 1:4.5 $\mathrm{f}=18 \mathrm{~cm}$ and Agfa ISS $21 \mathrm{DIN}$ emulsions operated together and provided plates covering slightly more than $50 \%$ of the visible sky. The total number of all plates exceeds 100,000 .

\section{CCD Monitoring Experiment (OTM - Optical Transient Monitor)}

\subsection{THE GOAL OF THE EXPERIMENT}

The aim of the experiment is to develop a reliable monitoring service at optical wavelengths and to solve the question whether or not the gamma ray bursts are accompanied by optical emissions. The detection of these phenomena at optical wavelengths could significantly help to answer the question of the physical nature of gamma ray bursters (still unknown). OTM represents a low cost automatic operation experiment with good time resolution, a medium sensitivity, low $(0.1$ deg.) angular resolution and excellent background elimination. A double CCD camera with wide field lenses will monitor $13 \%$ of the full sky hemisphere for optical transient phenomena. Two such systems are planned, separated by $100 \mathrm{~km}$ to be able to eliminate all effects in the earth atmosphere and near space. The experiment is also able to provide very wide-field monitoring of the sky for general use. For this purpose, the expected sensitivity is to detect stars and objects up to mag. 10 with an exposure of the 100 sec.

\subsection{THE EXPERIMENT PROPOSED AND TRIED}

The device to be developed represents a simple CCD wide-field camera with a lens of a short focal length. The idea is to develop a simple device but with a sophisticated system to exclude all false triggers. The previous experiments in this field have shown that the main problem of these investigations is the high signal to noise ratio and hence it is rather important to be able to eliminate reasonably all background and false triggers.

To do it, we suggest the construction of a double CCD camera with two independent paths equipped with different filters and to develop specialised software excluding false triggers and triggers caused by satellites. The basic parameters of the proposed device are summarized in the following table:
Lens
wide-field $\mathrm{f}=6 \mathrm{~mm}, 1: 16$

Focal detector

CCD camera ST-6 SBIG

Area of the focal detector $8.63 \times 6.53 \mathrm{~mm}, 375 \times 242$ pixels

Pixel size

$23 \times 27$ microns

Field of view

Angular resolution

$47 \times 55 \mathrm{deg}^{2}$ or $2606 \mathrm{deg}^{2}$ (12.6\% of the full sky hemisphere)

Limiting magnitude

$0.1 \mathrm{deg}$. No. of optical paths in one device: 2 .

Alternatively, other lenses and/or CCD arrays may be used, resulting in different parameters. There is only little experience with similar very wide-field systems in astronomy and so we intend 


\section{Optical Transient Monitor}

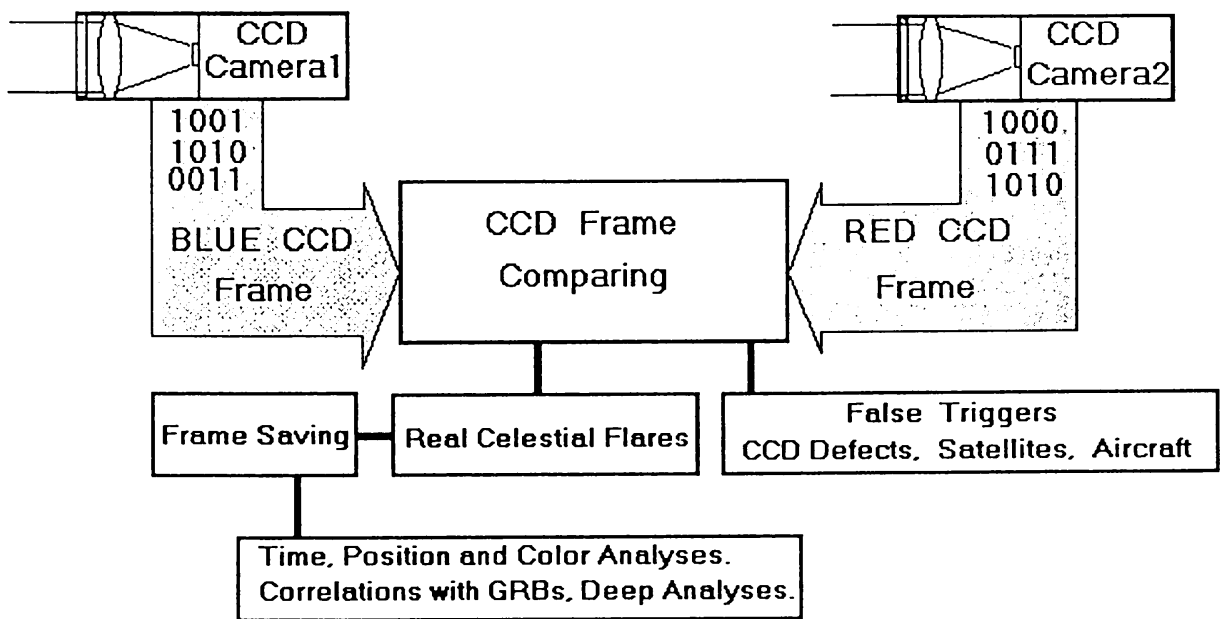

Figure 1. The proposed CCD monitoring configuration.

to test different lenses and CCD detectors carefully. The idea is to get very wide-field monitoring of the sky with still reasonable quality and parameters.

We propose the development of the new automatic working system for short optical light burst detections (counterparts of the x-ray and gamma-ray bursts) using the Texas Instruments Digital Signal Processor (DSP) TMS320C40. We would like to design a system based on the classical PC computer as host computer for this DSP processor. The TMS320C40 processor is available on the market and is delivered as the classical PC add-on cards. The PC computer as the host machine will serve this DSP processor with input data which will be necessary for its work. On the other side, the PC will receive results about parallel image data processing and will store these images on its harddisk if the optical burst will be detected. Using SANG Mega-Link-C40 PC card we will be able to exchange data between $P C$ and DSP processor with speeds up to $5 \mathrm{MB} / \mathrm{s}$. We would like to develop a user friendly interface for using DSP processor based on MSWindows 3.1 and OOP (Object Oriented Programming) technology. We have finished our new system for parallel ST6 CCD camera driving (up to 3 CCD cameras) now, but this system is not able to work in real time due to PC computer capabilities. This project named WinST6 is based on the OOP technology. We would like to work out our experiences with ST6 CCD cameras under MS-Windows to the DSP TMS320C40 processor using its $3 \mathrm{~L}$ parallel C language. All real time data acquisition and image data processing will be done on the DSP side. 

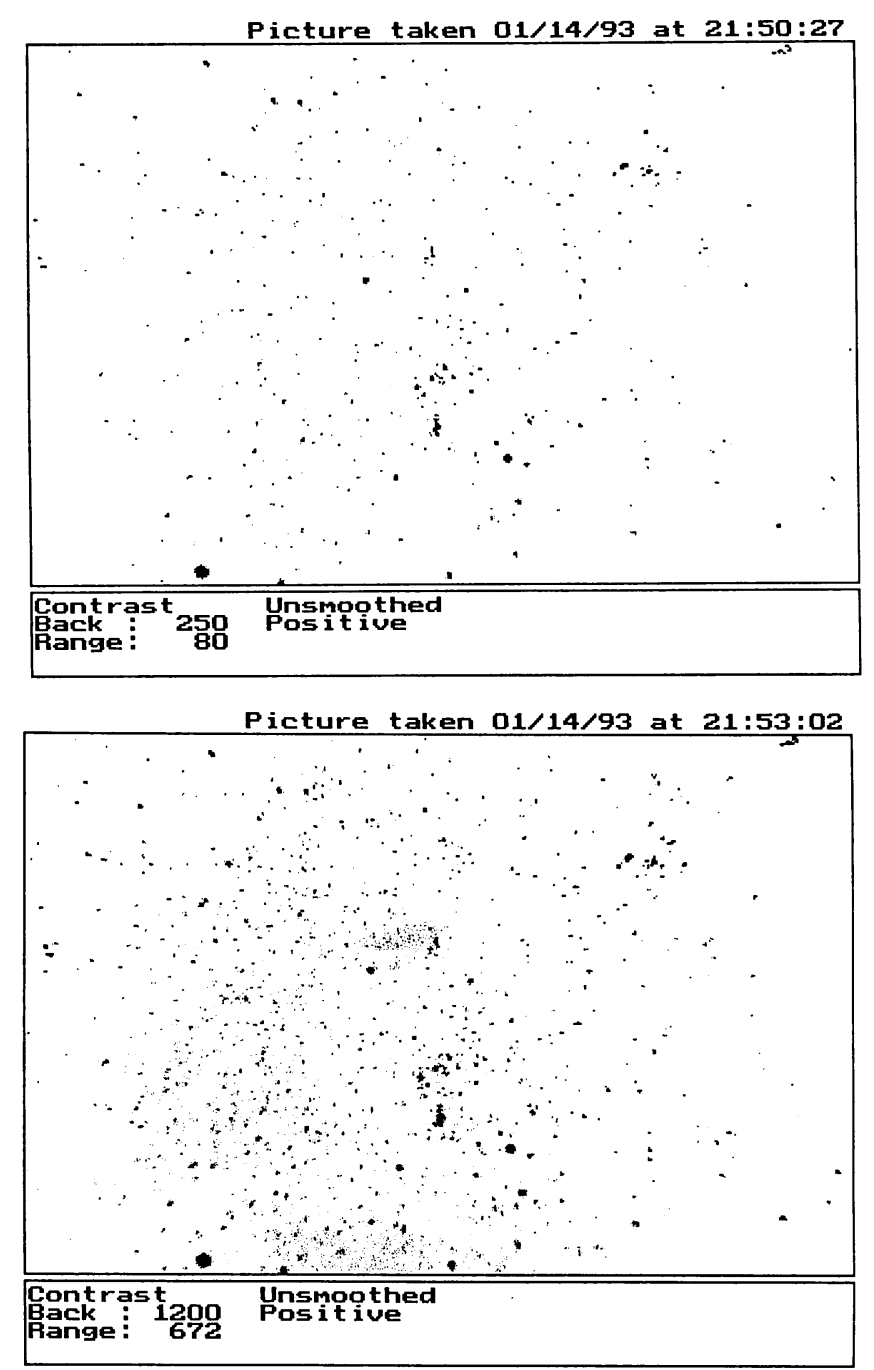

Figure 2. Test images of the Orion area obtained with slightly defocused $8.0 \mathrm{~mm}$ lens $(\mathrm{f}=8.0 \mathrm{~mm}, 1: 1.6)$ and SBIG ST-6 CCD camera. The field-of-view is roughly $48 \times 40 \mathrm{deg}^{2}$. (a) Exposure $1 \mathrm{sec}$. (b) Exposure 9 sec.

\subsection{HOW THE SYSTEM WORKS}

The suggested system consists of two or (better) four CCD cameras ${ }^{\dagger}$. They are on the same

${ }^{T}$ Two CCD cameras represents the minimal configuration. A significantly better time coverage can be achieved with $4 \mathrm{CCD}$ cameras. We consider this alternative further, however also the cheaper $(2 \mathrm{CCD}$ cameras) alternative is acceptable, at least for the first stage. 
mount and are centred on the same place on the sky. The centring will be done with faint mechanical screws and special software. Each camera is driven with one I/O port of the TMS320C40 via special interface which will be designed. These four cameras are divided into two subsets each consisting of two cameras. These two cameras work (exposure) always together. One camera into each subset has a red filter and the second camera the blue one. This configuration gives the following main features:

1) Real Time Data Acquisition.

2) Detector Electronics False Triggers Elimination.

3) Aircraft and Satellite Elimination. 